\title{
Research on Evaluation Emulsify Ability for Polyphenols in Vegetable Oils
}

\author{
JIANG Li-Qin ${ }^{1, a}$ \\ 1Department of Pharmacy, Zhejiang Chinese Medical University, Hangzhou, 310053,China \\ ajiang@zcmu.edu.cn
}

\begin{abstract}
Keywords: polyphenol, vegetable oil, emulsion, stability, evaluation
Abstract. The emulsification properties and emulsion stability of polyphenols on vegetable oils were investigated. Vegetable oil is always processed into foods existing as emulsions. In this study the author used edible oil-in-water emulsions stabilized by soya protein isolate (SPI) to investigate the differences between the emulsifying ability and stability of protein-stabilized emulsions. The author also investigated the interfacial activity of the edible oils, aglycon and glucoside of phenolic compound. From the results, virgin olive oil existed better emulsifying ability and stability than the other edible oils. Phenolic content, especially aglycon and glycoside compounds in olive oil were expected to responsible for the emulsification activity and emulsion stability of olive oil.
\end{abstract}

\section{Introduction}

Vegetable oils play important role in human life, including contributions of essential nutrients, energy, mouth-fell, flavor, palatability, emulsifying, and complex characteristics to food products[1-3]. Vegetable oils are obtained from beans or seeds by pressing and/or by solvent extraction. Oil seed varieties, extracting method, cultivars, etc., are responsible for the different from oil to oil[4]. The composition of vegetable oils have been known to be different due to cultivar, variety, maturation, rainfall, irrigation, processing method, and storage, etc[5,6].

Besides direct consuming, vegetable oils are always processed into foods existing as emulsions, such as dressing or mayonnaise etc. Proteins are commonly used in food products to facilitate the formation and improve the stability of oil-in-water emulsions[7,8]. Mixtures of proteins and oils can be found among the ingredient of a wide range of colloidal food systems ranging from soymilk to dressing. The stability of emulsion is responsible for oxidative stability as well as sensibility[7,9]. Therefore, analytical techniques are needed to provide information about the properties of emulsions.

Olive oil is well known to contain phenolic compounds. These compounds may be surface active because of their amphiphilic structure, facilitating emulsification when olive oil is mixed with water. The objective of this study is to investigate the emulsifying properties of various vegetable oils,including olive oil. As emulsifiers, soya protein isolate (SPI) was used.

\section{Materials and Methods}

\section{Materials}

Rapeseed oil, virgin olive oil, sesame oil, and soybean oil were purchased from market. Four kinds of virgin olive oils cv. Manzanillo, Lucca, Nevadillo Blanco and Mission, were prepared with continuous two-phase centrifuge olive oil processing plant (Oliomio 50, Toscana Enologica Mori, Italy) from random picked olive fruits from Uchinomi Town Olive Garden Public Co., Shodo Island, Japan.

\section{Reagents}

SPI was obtained from Fuji Oil Manufactory (Osala, Japan). Rutin and $n$-tetradecane were obtained from Wako Pure Chemical Industries (Osaka, Japan). Quercetin was obtained from Extrasynthese (Genay, France). 


\section{Emulsion preparation}

SPI was dissolved in distilled water, and oil sample was added. The mixture was vortexed for 10 sec, then submitted to homogenize for $2 \mathrm{~min}$ in a high-speed blender (Nichion Irikagakukiki Seisakusho, Tokyo, Japan) operated at 22,000 rpm. The emulsion was kept in $20^{\circ} \mathrm{C}$ until analysis.

\section{Particle size distribution measurement}

A laser diffraction particle size analyzer (model LA-500, Horiba Seisakusho, Kyoto, Japan) was used to determine the droplet-size distribution.

\section{Interfacial tension measurement}

Interfacial tension at the oil-water interface was measured with a Wilhelmy plate type of surface tensiometer (Model CBVP-A3; Kyowa Kagaku Co., Tokyo, Japan) by using a platinum plate (24 mm $\times 10 \mathrm{~mm}$ ) and is expressed as dyne $/ \mathrm{cm}$. The oil phase used for measuring the interfacial properties was $n$-tetradecane.

\section{Results and Discussion}

It is generally accepted that SPI is poor-emulsifying proteins as compared with caseins and milk whey proteins[10]. Major component of SPI were glycinin and $\beta$-conglycinin, which were gigantic subunit proteins. Because of large size and subunit structure, the diffusion and the adsorption of these soy proteins onto the interface are thought to be difficult. The use of the poor-emulsifying proteins may magnify the difference in emulsion properties among various olive oils varying in phenolic compounds.

\section{Emulsions stabilized by SPI}

Olive and vegetable oils were emulsified by SPI and the particle size-distribution of the emulsions was measured just after preparation. The results on two olive oils, Manzanillo and Lucca virgin olive oils, and the commercial rapeseed oil were presented in Fig. 1.

The main particle size of emulsions including the olive oils was in the range of 1-10 $\mu \mathrm{m}$. However, the main particle size of the rapeseed oil-in-water emulsion was more distributed and shifted to the larger direction, that is, in the range 1-100 $\mu \mathrm{m}$. Furthermore, the particle size distribution was bimodal, whereas the distribution curve of the olive oil-in water emulsions showed the single peak. This suggests the good emulsifying properties of the olive oil as compared to those of the rapeseed oil.

Fig. 2. shows the results of distribution curves of all the emulsions just after preparation. The results were presented in the cumulative form for the easy comparison. The upper and lower figures indicate the results in the short range $(0-40 \mu \mathrm{m}$ in the normal scale) and the long range $(0.1-200 \mu \mathrm{m}$ in the logarithmic scale), respectively. In the long range, the difference in the distribution patterns among the emulsions were difficult to be observed, but the expansion of scale enabled us to compare the distributions of emulsions more clearly. The distribution curves of Manzanillo and Lucca virgin olive oil reached $100 \%$ in smaller diameter values, indicating the formation of fine emulsion particles. On the other hand, the curve of Mission virgin olive oil reached $100 \%$ above $10 \mu \mathrm{m}$, indicating the formation of coarse emulsion particles. In other words, emulsification properties of Manzanillo and Lucca virgin olive oil were superior to those of Mission virgin olive oil. The emulsifying properties of soybean oil and rapeseed oil were less than those of Manzanillo or Lucca but better than those of Mission olive oil. 


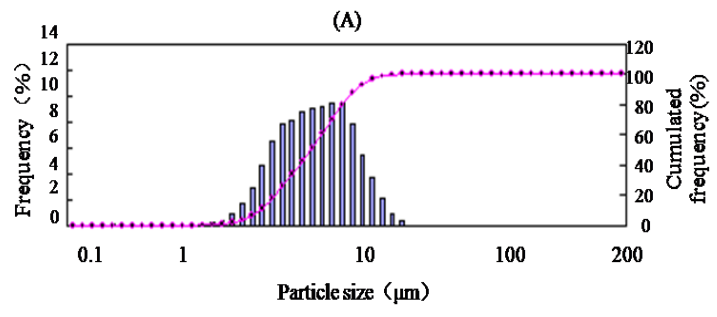

(B)

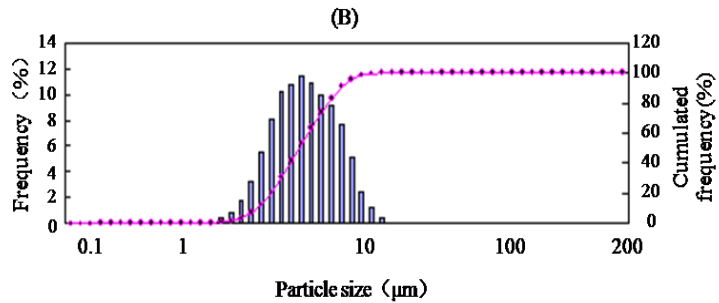

(C)

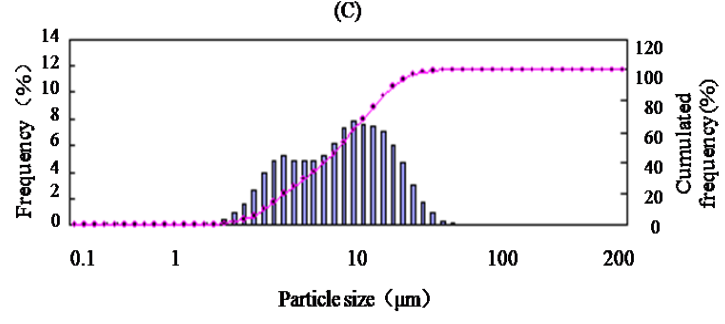

Fig. 1. Particle size-distribution of the emulsions prepared using ISP.

The oils used were (A) Manzanillo virgin olive oil, (B) Lucca virgin olive oil, (C) Rapeseed oil.

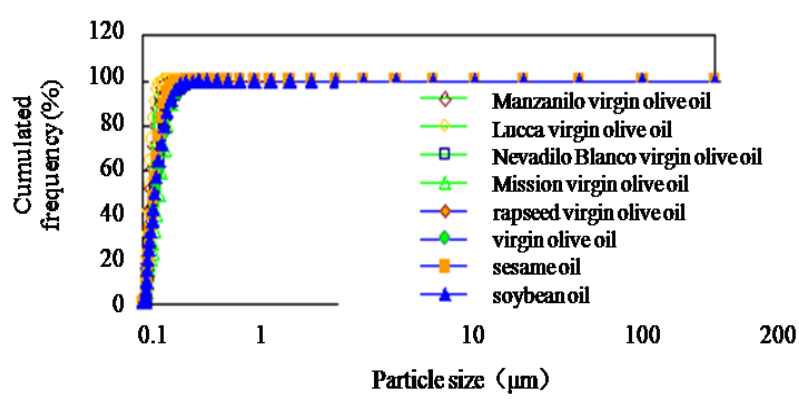

(B)

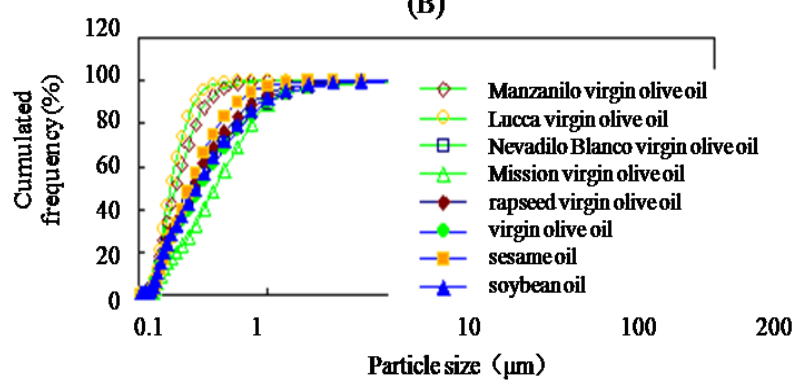

Fig. 2. Particle size-distribution of the emulsions prepared using SPI. The oils used were (A) particle size 0-200 $\mu \mathrm{m}$ (B) particle size 0-45 $\mu \mathrm{m}$.

Emulsion stability was assessed by measuring the oil droplet size distributions of emulsions for three consecutively days. Fig. 3 shows the results of emulsions of Manzanillo virgin olive oil and rapeseed oil. The pattern of Manzanillo emulsions was changed to the bimodal distribution on second third days, but extremely large particles above $100 \mu \mathrm{m}$ were scarcely observed. On the other hand, the large particles with about $200 \mu \mathrm{m}$ diameter were formed on third day in the case of rapeseed oil emulsion. The creaming was also observed clearly in this emulsion (data not shown). These results suggest that the emulsion stability for Manzanillo virgin olive oil was higher than that for rapeseed oil. The emulsion of Lucca olive oil also demonstrated the high stability (data not shown).
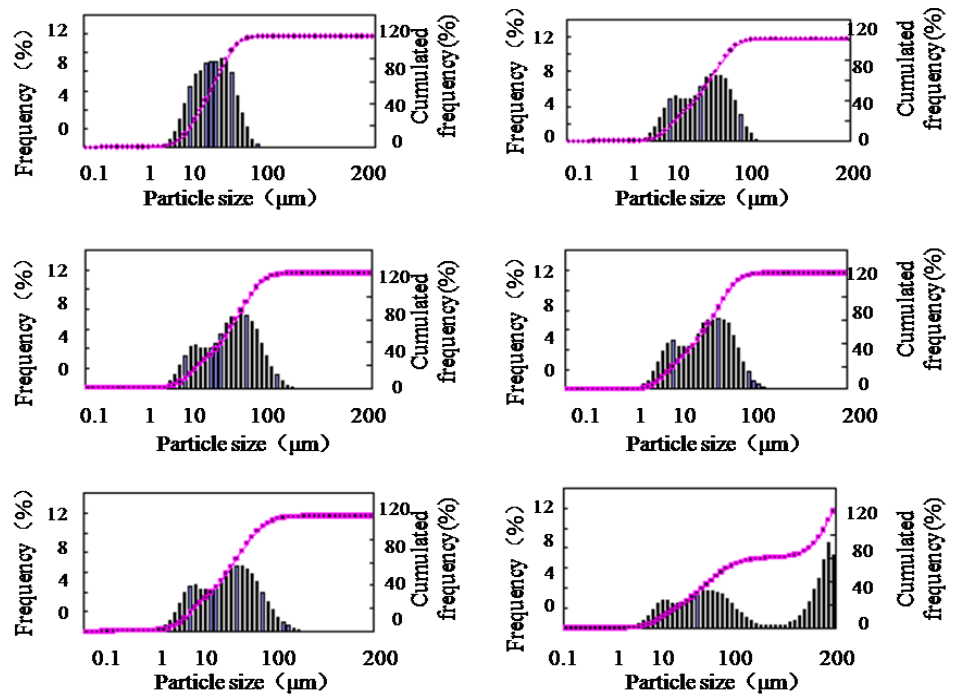

Fig. 3. Oil droplet size distributions of emulsions prepared using SPI for three consecutively days. (A) Manzanillo olive oil, (B) rapeseed oil. 


\section{Conclusions}

The emulsification properties and emulsion stability of various olive oils from Shodo Island were investigated. From the result, emulsions stabilized by SPI presented good emulsifying properties than that stabilized by OVA. Among the olive oils, Manzanillo and Lucca olive oil presented better emulsification property compared with other olive oils and vegetable oils. The time-dependent interfacial tension of an aglycon (quercetin) and a glycoside (rutin) was different. Aglycon and glycoside compound in olive oil is expected to responsible for the emulsification activity and emulsion stability of olive oils.

\section{Acknowledgements}

This work was financially supported by the Public Welfare Technology Application Research in Zhejiang province (2014C32115) and Professional Development Program of University Visiting Scholar (FX2014029)

\section{References}

[1] Nawar, W. W. Lipids. In “Food Chemistry.” 3 ed. (Marcel Dekker, New York, USA 1996)

[2] Van Ruth, S.M. King C. and Giannouli, P: J. Agric. Food Chem. Vol.50 (2002), p. 2365-2371.

[3] Witting, L.P. and Dimick, P.S. Effects of processing on food lipids. In "Handbook of nutritive value of processed food, volume (I) food for human use.” Ed. Rechcigal, M., Jr. (CRC press, USA 1982)

[4] Boskou, D. Olive oil. In "Vegetable oils in food technology composition, properties and uses.”(CRC Press, Blackwell, Boca Raton, FL, USA 2002)

[5] Fustier P, Achouri A, Taherian AR, et al., J Agric Food Chem. Vol.63(2015), p.9239-9250.

[6] Chiu N, Hewson L, Fisk I, et al.: Food Funct. Vol. 6 (2015), p.1428-1434.

[7] Mónica Obando, Antonios Papastergiadis, Shanshan Li,et al.: J. Agric. Food Chem., Vol.63 (2015), p. 9820-9830.

[8] Prak K, Naka M, Tandang-Silvas MR.: Protein Eng Des Sel. Vol. 28 (2015), p. 281-291.

[9] Onsaard E, Putthanimon J, Singthong, J.: Food Sci Technol Int. Vol. 20 (2014), p. 617-628.

[10] Matsumura, Y. :Journal of the Japanese Society for Food Science and Technology.Vol. 2(2001), 87-95 (in Japanese) 